CRYSTALLOGRAPHIC COMMUNICATIONS

ISSN 2056-9890

Received 31 March 2021

Accepted 4 May 2021

Edited by D. Chopra, Indian Institute of Science Education and Research Bhopal, India

Keywords: crystal structure; anthracene; C$\mathrm{H} \cdots \pi$ interactions; $\pi-\pi$ stacking interactions.

CCDC reference: 2072800

Supporting information: this article has supporting information at journals.iucr.org/e

\section{Crystal structure of 4-(anthracen-9-yl)pyridine}

\author{
Meng Zhao, Gang Zhang, Jingmiao Zhang, Shan Huang, Xiuxia Liu and Fei Li*
}

Department of Nuclear Medicine, the Second Hospital of Anhui Medical University, Hefei 230601, People's Republic of China. *Correspondence e-mail: lifei007@139.com

The title compound, $\mathrm{C}_{19} \mathrm{H}_{13} \mathrm{~N}$, which crystallizes in the monoclinic $\mathrm{C} 2 / \mathrm{c}$ space group with one half-molecule in the asymmetric unit, was synthesized by Suzuki-Miyaura cross-coupling reaction of 9-bromoanthracene with pyridin-4ylboronic acid and purified by column chromatography on silica gel. Lightyellow crystals of 4-(anthracen-9-yl)-pyridine suitable for X-ray diffraction were collected by the solvent evaporation method. In the crystal, pairs of molecules are connected by intermolecular $\mathrm{C}-\mathrm{H} \cdots \pi$ (pyridine) interactions $[d(\mathrm{H} 7 \cdots C g)$ $=2.7391(2) \AA]$, forming cyclic centrosymmetric dimers, further resulting in an infinite one-dimensional linear chain along the $c$-axis direction. Weak face-toface $\pi-\pi$ stacking interactions $[d(C g \cdots C g)=3.6061(2) \AA]$ link neighboring lamellar networks into the supramolecular structure.

\section{Chemical context}

Anthracene and its derivatives constitute a very famous class of fluorophores that have been widely used in the development of functional fluorescent chemosensors because of their intriguing photophysical properties and chemical stability (Martínez-Máñez et al., 2003). One of the most important steps in the rational molecule design of anthracene-based chemosensors is the judicious combination with functional chemical recognition moieties, which can be used for monitoring and quantifying of abnormal physiological changes at the subcellular level (Densil et al., 2018; Mondal et al., 2014; Anand et al., 2015; Shree et al., 2019). It has been found that 9,10-distyrylanthracene derivatives with restricted intramolecular rotations often lead to aggregation-induced emission characteristics (Lu et al., 2010). In recent years, there has been an increased effort to combine anthracene derivatives with $\mathrm{N}$ - or O-coordinated single ligands and other attractive mixed ligands in order to construct tunable fluorescent ligands (Dey et al., 2016; Yao et al., 2019). As part of our studies in this area, we report herein the synthesis and crystal structure of a fluorescent monopyridine ligand, $\mathrm{C}_{19} \mathrm{H}_{13} \mathrm{~N}$.

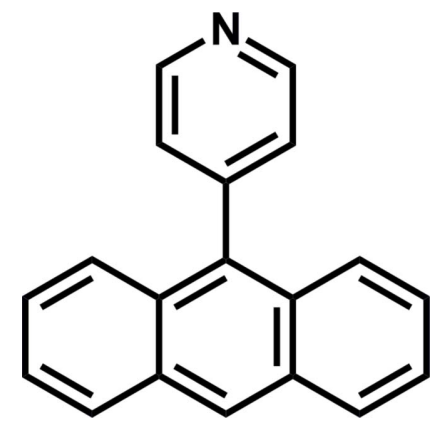




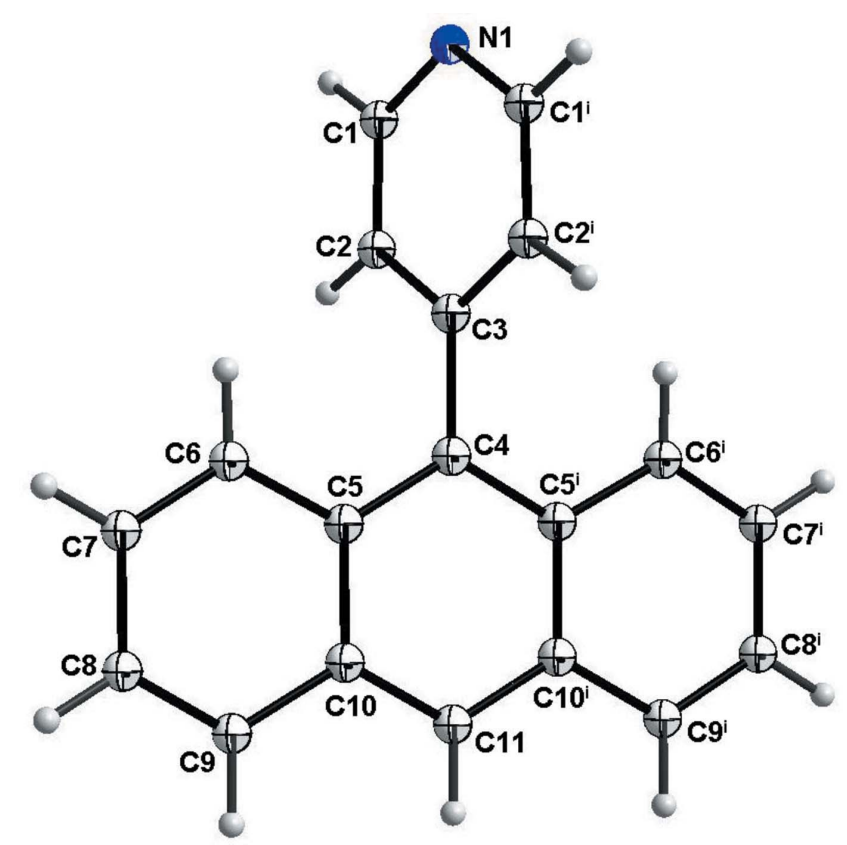

Figure 1

The molecular structure of 4-(anthracen-9-yl)-pyridine with displacement ellipsoids at the $50 \%$ probability level. Symmetry code: (i) $-x, y, \frac{1}{2}-z$.

\section{Structural commentary}

As shown in Fig. 1, single-crystal X-ray diffraction analysis reveals that 4-(anthracen-9-yl)-pyridine crystallizes in the monoclinic $C 2 / c$ space group with half molecule in the asymmetric unit (Table 1). In the structure of the title compound, the $\mathrm{C}-\mathrm{C}$ bond lengths of the benzene ring range from 1.3534 (13) to 1.4352 (1) $\AA$, and the $\mathrm{C}-\mathrm{N}$ bond length is 1.3351 (11), which is comparable with the literature reported (Zhao et al., 2016). The bond angle of $\mathrm{N} 1-\mathrm{C} 1-\mathrm{C} 2$ is $124.161(7)^{\circ}$, closed to the ideal bond angle of $120^{\circ}$ for benzene ring. The pyridine ring is inclined to the benzene ring at a dihedral angle of $71.64(4)^{\circ}$.

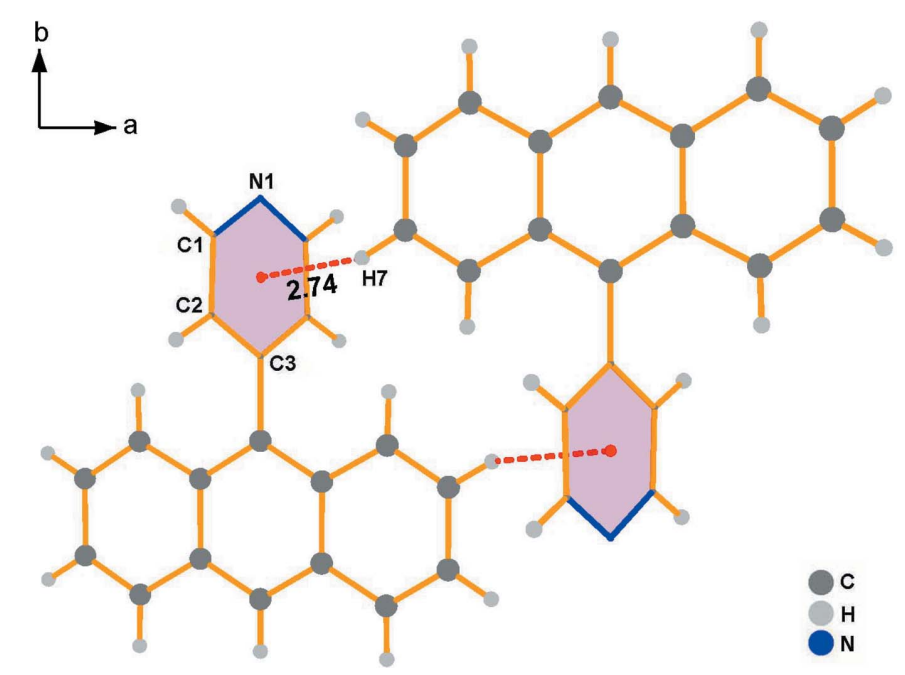

Figure 2

The hydrogen-bonded centrosymmetric dimer along the $c$ axis. Dashed lines indicate $\mathrm{C}-\mathrm{H} \cdots \pi$ interactions.
Table 1

Hydrogen-bond geometry $\left(\AA,^{\circ}\right)$.

$C g$ is the centroid of the pyridine ring.

\begin{tabular}{lllll}
\hline$D-\mathrm{H} \cdots A$ & $D-\mathrm{H}$ & $\mathrm{H} \cdots A$ & $D \cdots A$ & $D-\mathrm{H} \cdots A$ \\
\hline $\mathrm{C} 7-\mathrm{H} 7 \cdots C g^{\mathrm{i}}$ & 0.93 & 2.74 & $3.5606(12)$ & 148 \\
$\mathrm{C} 7-\mathrm{H} 7 \cdots C g^{\mathrm{ii}}$ & 0.93 & 2.74 & $3.5606(12)$ & 148 \\
\hline
\end{tabular}

Symmetry codes: (i) $-x+1,-y+1,-z+1$; (ii) $x+1,-y+1, z+\frac{1}{2}$.

\section{Supramolecular features}

In the crystal, the hydrogen atom of anthracene ring contributes to the formation of a $\mathrm{C} 7-\mathrm{H} 7 \cdots \pi$ contact with the pyridine ring (Table 1); the resulting cyclic centrosymmetric dimer is shown in Fig. 2. Subsequently, the paired C$\mathrm{H} \cdots \pi$ (pyridine) hydrogen-bonding interactions connect neighboring dimers, resulting in an infinite 1-D linear chain (Fig. 3), which is basis for extension of the dimensionality. As shown in Figs. 4 and 5, the crystal packing involves weak faceto-face $\pi-\pi$ stacking interactions $[d(C g \cdots C g)=3.6095(7) \AA]$ between two benzene rings related by the symmetry operation $1-x, y, \frac{1}{2}-z$.

\section{Database survey}

A search in the Cambridge Structural Database (CSD, Version 5.41, update March 2021; Groom et al. 2016) revealed that this is the first example of a structurally characterized 4-(anthracen-9-yl)-pyridine. At the same time, a CSD search for compounds containing the 4-(anthracen-9-yl)-pyridine substructure identified only one compound, viz. $\mathrm{Ag}_{12}\left(\mathrm{SCH}_{2} \mathrm{C}_{6} \mathrm{H}_{5}\right)_{6}\left(\mathrm{CF}_{3} \mathrm{COO}\right)_{6}\left(L_{4}\right)_{6}\left[L_{4}=4\right.$-(anthracen-9-yl)pyridine; Li et al., 2018] in which the pyridine ring of this compound is inclined to the benzene ring at a dihedral angle of $73.28^{\circ}$.

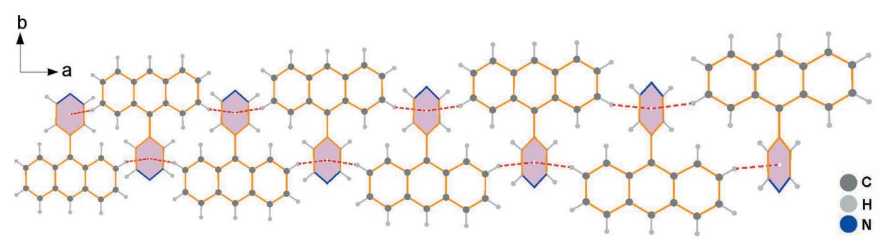

Figure 3

View of the 1-D chain-like structure of the title compound along the $c$ axis.

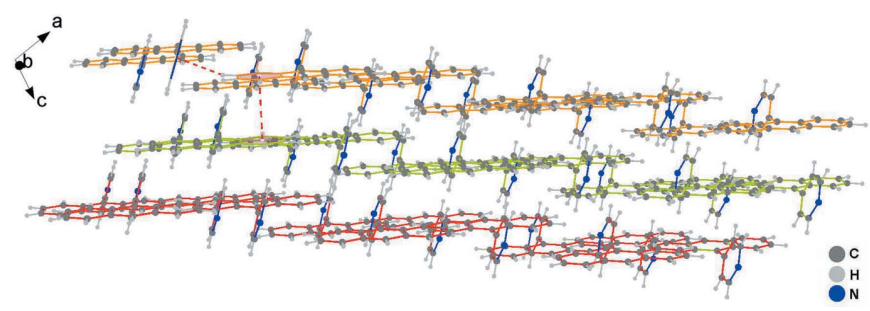

Figure 4

Crystal packing projected via $\mathrm{C}-\mathrm{H} \cdots \pi$ and $\pi-\pi$ stacking interactions. 


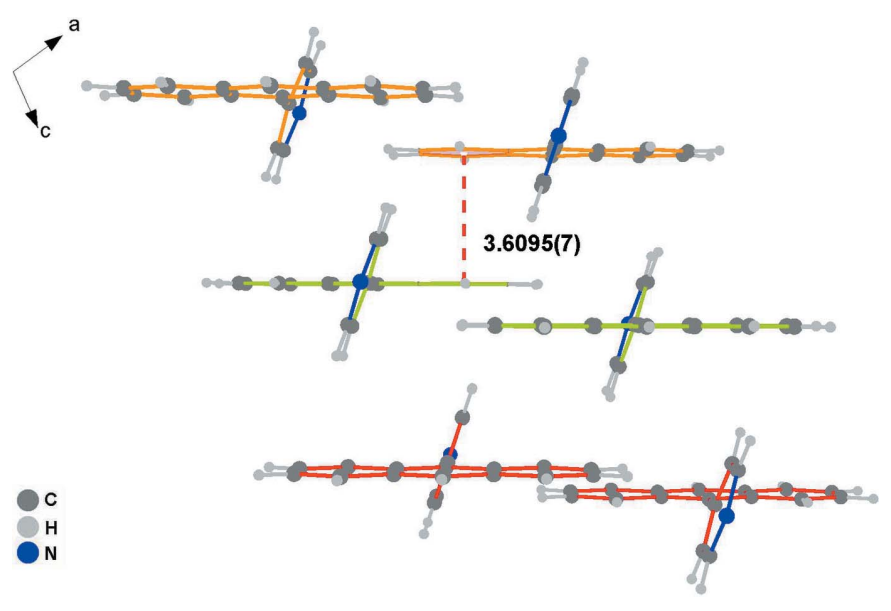

Figure 5

$\pi-\pi$ stacking interactions of molecules in the crystal structure of the title compound.

\section{Synthesis and crystallization}

4-(Anthracen-9-yl)-pyridine was synthesized by the SuzukiMiyaura cross-coupling reaction according to a previously reported protocol (Zhao et al., 2019). As shown in Fig. 6, under a nitrogen atmosphere, 9-bromoanthracene (2.56 g, $10 \mathrm{mmol})$, pyridin-4-ylboronic acid (1.23 g, $10 \mathrm{mmol})$ and tetratriphenyl phosphine palladium $(0.10 \mathrm{~g}, 0.1 \mathrm{mmol})$ were dissolved in toluene $(90 \mathrm{~mL})$ followed by the addition of potassium carbonate aqueous solution $(22 \mathrm{wt} \%, 40 \mathrm{~mL})$ under constant stirring. The reaction mixture was subsequently refluxed for $12 \mathrm{~h}$, and the mixture was then further purified by column chromatography using petroleum/ethyl acetate $(3: 1, v / v)$ as eluent to give yellow solid 4-(anthracen-9-yl)pyridine (1.76 g, yield $69 \%$ ).

Crystals of 4-(anthracen-9-yl)-pyridine suitable for X-ray analysis were obtained by the solvent evaporation method. In detail, solid 4-(anthracen-9-yl)-pyridine (0.013 g, $0.05 \mathrm{mmol})$ was dissolved in $0.5 \mathrm{~mL}$ of dichloromethane and $5 \mathrm{~mL}$ of ethyl acetate. The mixture solvent was evaporated slowly at room temperature for about 2 weeks. Light-yellow crystals of 4-(anthracen-9-yl)-pyridine suitable for X-ray diffraction were collected.

\section{Refinement}

Crystal data, data collection and structure refinement details are summarized in Table 2 . $\mathrm{H}$ atoms were positioned geome-

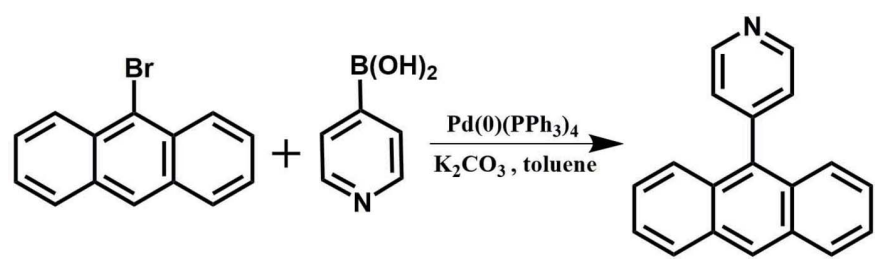

Figure 6

Synthesis of 4-(anthracen-9-yl)-pyridine.
Table 2

Experimental details.

\begin{tabular}{|c|c|}
\hline \multicolumn{2}{|l|}{ Crystal data } \\
\hline Chemical formula & $\mathrm{C}_{19} \mathrm{H}_{13} \mathrm{~N}$ \\
\hline$M_{\mathrm{r}}$ & 255.30 \\
\hline Crystal system, space group & Monoclinic, $C 2 / c$ \\
\hline Temperature $(\mathrm{K})$ & 296 \\
\hline$a, b, c(\AA)$ & $\begin{array}{l}6.0777(4), 20.9211(16) \\
\quad 10.2574(7)\end{array}$ \\
\hline$\beta\left(^{\circ}\right)$ & $102.476(3)$ \\
\hline$V\left(\AA^{3}\right)$ & $1273.45(16)$ \\
\hline$Z$ & 4 \\
\hline Radiation type & Mo $K \alpha$ \\
\hline$\mu\left(\mathrm{mm}^{-1}\right)$ & 0.08 \\
\hline Crystal size $(\mathrm{mm})$ & $0.18 \times 0.15 \times 0.13$ \\
\hline \multicolumn{2}{|l|}{ Data collection } \\
\hline Diffractometer & Bruker APEXII CCD \\
\hline Absorption correction & $\begin{array}{l}\text { Multi-scan (SADABS; Krause et } \\
\quad \text { al., 2015) }\end{array}$ \\
\hline$T_{\min }, T_{\max }$ & $0.699,0.745$ \\
\hline $\begin{array}{l}\text { No. of measured, independent and } \\
\text { observed }[I>2 \sigma(I)] \text { reflections }\end{array}$ & $4703,1144,1022$ \\
\hline$R_{\text {int }}$ & 0.029 \\
\hline$(\sin \theta / \lambda)_{\max }\left(\AA^{-1}\right)$ & 0.602 \\
\hline \multicolumn{2}{|l|}{ Refinement } \\
\hline$R\left[F^{2}>2 \sigma\left(F^{2}\right)\right], w R\left(F^{2}\right), S$ & $0.036,0.105,1.11$ \\
\hline No. of reflections & 1144 \\
\hline No. of parameters & 94 \\
\hline $\mathrm{H}$-atom treatment & $\mathrm{H}$-atom parameters constrained \\
\hline$\Delta \rho_{\max }, \Delta \rho_{\min }\left(\mathrm{e} \AA^{-3}\right)$ & $0.15,-0.14$ \\
\hline
\end{tabular}

Computer programs: APEX3 (Bruker, 2018), SAINT (Bruker, 2015), SHELXT (Sheldrick, 2015a), SHELXL (Sheldrick, 2015b) and OLEX2 (Dolomanov et al., 2009).

trically $(\mathrm{C}-\mathrm{H}=0.93 \AA)$ and refined as riding with $\mathrm{U}_{\text {iso }}(\mathrm{H})=$ $1.2 \mathrm{U}_{\mathrm{eq}}(\mathrm{C})$.

\section{Funding information}

This work was supported by grants from the National Natural Science Foundation of China Incubation Program of the Second Hospital of Anhui Medical University (2020GQFY04) and Anhui Medical University Research Fund (2020xkj024).

\section{References}

Anand, T., Sivaraman, G., Mahesh, A. \& Chellappa, D. (2015). Anal. Chim. Acta, 853, 596-601.

Bruker (2015). SAINT. Bruker AXS Inc., Madison, Wisconsin, USA. Bruker (2018). APEX3. Bruker AXS Inc., Madison, Wisconsin, USA.

Densil, S., Chang, C. H., Chen, C. L., Mathavan, A., Ramdass, A., Sathish, V., Thanasekaran, P., Li, W. S. \& Rajagopal, S. (2018). Luminescence, 33, 780-789.

Dey, B., Mondal, R. K., Dhibar, S., Chattopadhyay, A. P. \& Bhattacharya, S. C. (2016). J. Lumin. 172, 1-6.

Dolomanov, O. V., Bourhis, L. J., Gildea, R. J., Howard, J. A. K. \& Puschmann, H. (2009). J. Appl. Cryst. 42, 339-341.

Groom, C. R., Bruno, I. J., Lightfoot, M. P. \& Ward, S. C. (2016). Acta Cryst. B72, 171-179.

Krause, L., Herbst-Irmer, R., Sheldrick, G. M. \& Stalke, D. (2015). J. Appl. Cryst. 48, 3-10.

Li, Y. L., Zhang, W. M., Wang, J., Tian, Y., Wang, Z. Y., Du, C. X., Zang, S. Q. \& Mak, T. C. W. (2018). Dalton Trans. 47, 14884-14888.

Lu, H., Xu, B., Dong, Y., Chen, F., Li, Y., Li, Z., He, J., Li, H. \& Tian, W. (2010). Langmuir, 26, 6838-6844.

Martínez-Máñez, R. \& Sancenón, F. (2003). Chem. Rev. 103, 44194476. 
Mondal, B. \& Kumar, V. (2014). RSC Adv. 4, 61944-61947.

Sheldrick, G. M. (2015a). Acta Cryst. A71, 3-8.

Sheldrick, G. M. (2015b). Acta Cryst. C71, 3-8.

Shree, G. J., Sivaraman, G., Siva, A. \& Chellappa, D. (2019). Dyes Pigments, 163, 204-212.
Yao, S. L., Liu, S. J., Tian, X. M., Zheng, T. F., Cao, C., Niu, C. Y., Chen, Y. Q., Chen, J. L., Huang, H. \& Wen, H. R. (2019). Inorg. Chem. 58, 3578-3581.

Zhao, M., Gao, Y., Ye, S., Ding, J., Wang, A., Li, P. \& Shi, H. (2019). Analyst, 144, 6262-6269.

Zhao, M., Tan, J., Su, J., Zhang, J., Zhang, S., Wu, J. \& Tian, Y. (2016). Dyes Pigments, 130, 216-225. 


\section{supporting information}

Acta Cryst. (2021). E77, 605-608 [https://doi.org/10.1107/S2056989021004710]

\section{Crystal structure of 4-(anthracen-9-yl)pyridine}

\section{Meng Zhao, Gang Zhang, Jingmiao Zhang, Shan Huang, Xiuxia Liu and Fei Li}

Computing details

Data collection: APEX3 (Bruker, 2018); cell refinement: SAINT (Bruker, 2015); data reduction: SAINT (Bruker, 2015); program(s) used to solve structure: SHELXT (Sheldrick, 2015a); program(s) used to refine structure: SHELXL (Sheldrick, 2015b); molecular graphics: OLEX2 (Dolomanov et al., 2009); software used to prepare material for publication: OLEX2 (Dolomanov et al., 2009).

4-(Anthracen-9-yl) pyridine

Crystal data

$\mathrm{C}_{19} \mathrm{H}_{13} \mathrm{~N}$

$M_{r}=255.30$

Monoclinic, $C 2 / \mathrm{c}$

$a=6.0777$ (4) $\AA$

$b=20.9211(16) \AA$

$c=10.2574$ (7) $\AA$

$\beta=102.476(3)^{\circ}$

$V=1273.45(16) \AA^{3}$

$Z=4$

Data collection

Bruker APEXII CCD diffractometer $\varphi$ and $\omega$ scans

Absorption correction: multi-scan

(SADABS; Krause et al., 2015)

$T_{\min }=0.699, T_{\max }=0.745$

4703 measured reflections

\section{Refinement}

Refinement on $F^{2}$

Least-squares matrix: full

$R\left[F^{2}>2 \sigma\left(F^{2}\right)\right]=0.036$

$w R\left(F^{2}\right)=0.105$

$S=1.11$

1144 reflections

94 parameters

0 restraints
$F(000)=536$

$D_{\mathrm{x}}=1.332 \mathrm{Mg} \mathrm{m}^{-3}$

Mo $K \alpha$ radiation, $\lambda=0.71073 \AA$

Cell parameters from 2979 reflections

$\theta=3.7-25.3^{\circ}$

$\mu=0.08 \mathrm{~mm}^{-1}$

$T=296 \mathrm{~K}$

Bulk, light yellow

$0.18 \times 0.15 \times 0.13 \mathrm{~mm}$

1144 independent reflections

1022 reflections with $I>2 \sigma(I)$

$R_{\text {int }}=0.029$

$\theta_{\max }=25.3^{\circ}, \theta_{\min }=3.7^{\circ}$

$h=-7 \rightarrow 6$

$k=-22 \rightarrow 24$

$l=-12 \rightarrow 12$

Hydrogen site location: inferred from neighbouring sites

$\mathrm{H}$-atom parameters constrained

$w=1 /\left[\sigma^{2}\left(F_{\mathrm{o}}^{2}\right)+(0.0556 P)^{2}+0.3319 P\right]$

where $P=\left(F_{\mathrm{o}}^{2}+2 F_{\mathrm{c}}{ }^{2}\right) / 3$

$(\Delta / \sigma)_{\max }<0.001$

$\Delta \rho_{\max }=0.15 \mathrm{e}^{-3}$

$\Delta \rho_{\min }=-0.14$ e $\AA^{-3}$ 


\section{Special details}

Geometry. All esds (except the esd in the dihedral angle between two 1.s. planes) are estimated using the full covariance matrix. The cell esds are taken into account individually in the estimation of esds in distances, angles and torsion angles; correlations between esds in cell parameters are only used when they are defined by crystal symmetry. An approximate (isotropic) treatment of cell esds is used for estimating esds involving l.s. planes.

Fractional atomic coordinates and isotropic or equivalent isotropic displacement parameters $\left(\AA^{2}\right)$

\begin{tabular}{lllll}
\hline & $x$ & $y$ & $z$ & $U_{\text {iso }} * / U_{\text {eq }}$ \\
\hline N1 & 0.000000 & $0.36240(5)$ & 0.250000 & $0.0360(4)$ \\
C1 & $0.12815(18)$ & $0.39626(5)$ & $0.18480(11)$ & $0.0359(3)$ \\
H1 & 0.219495 & 0.374032 & 0.138423 & $0.043^{*}$ \\
C2 & $0.13369(17)$ & $0.46237(5)$ & $0.18183(10)$ & $0.0324(3)$ \\
H2 & 0.226472 & 0.483317 & 0.134422 & $0.039^{*}$ \\
C3 & 0.000000 & $0.49724(7)$ & 0.250000 & $0.0266(4)$ \\
C4 & 0.000000 & $0.56870(6)$ & 0.250000 & $0.0267(4)$ \\
C5 & $0.18833(15)$ & $0.60220(5)$ & $0.32231(9)$ & $0.0279(3)$ \\
C6 & $0.38307(17)$ & $0.57083(5)$ & $0.39762(10)$ & $0.0308(3)$ \\
H6 & 0.387809 & 0.526405 & 0.399226 & $0.037^{*}$ \\
C7 & $0.56176(18)$ & $0.60434(5)$ & $0.46706(11)$ & $0.0357(3)$ \\
H7 & 0.686262 & 0.582594 & 0.515636 & $0.043^{*}$ \\
C8 & $0.56061(19)$ & $0.67201(5)$ & $0.46633(12)$ & $0.0405(3)$ \\
H8 & 0.683709 & 0.694491 & 0.514440 & $0.049^{*}$ \\
C9 & $0.38025(19)$ & $0.70397(5)$ & $0.39555(11)$ & $0.0407(4)$ \\
H9 & 0.381857 & 0.748415 & 0.394805 & $0.049^{*}$ \\
C10 & $0.18747(17)$ & $0.67085(5)$ & $0.32198(10)$ & $0.0325(3)$ \\
C11 & 0.000000 & $0.70319(7)$ & 0.250000 & $0.0371(4)$ \\
H11 & 0.000002 & 0.747643 & 0.250003 & $0.045^{*}$ \\
& & & &
\end{tabular}

Atomic displacement parameters $\left(\AA^{2}\right)$

\begin{tabular}{lllllll}
\hline & $U^{11}$ & $U^{22}$ & $U^{33}$ & $U^{12}$ & $U^{13}$ & $U^{23}$ \\
\hline $\mathrm{N} 1$ & $0.0410(8)$ & $0.0233(7)$ & $0.0417(7)$ & 0.000 & $0.0045(6)$ & 0.000 \\
$\mathrm{C} 1$ & $0.0393(6)$ & $0.0281(6)$ & $0.0414(6)$ & $0.0039(4)$ & $0.0110(5)$ & $-0.0046(4)$ \\
$\mathrm{C} 2$ & $0.0351(6)$ & $0.0280(6)$ & $0.0363(6)$ & $-0.0016(4)$ & $0.0123(4)$ & $-0.0006(4)$ \\
$\mathrm{C} 3$ & $0.0274(7)$ & $0.0243(7)$ & $0.0269(7)$ & 0.000 & $0.0026(5)$ & 0.000 \\
C4 & $0.0323(8)$ & $0.0235(8)$ & $0.0262(7)$ & 0.000 & $0.0108(5)$ & 0.000 \\
C5 & $0.0328(6)$ & $0.0261(6)$ & $0.0268(6)$ & $-0.0010(4)$ & $0.0110(4)$ & $-0.0001(3)$ \\
C6 & $0.0351(6)$ & $0.0260(6)$ & $0.0320(6)$ & $-0.0004(4)$ & $0.0085(4)$ & $0.0000(4)$ \\
C7 & $0.0333(6)$ & $0.0362(7)$ & $0.0364(6)$ & $-0.0006(4)$ & $0.0050(4)$ & $0.0003(4)$ \\
C8 & $0.0391(7)$ & $0.0359(7)$ & $0.0443(7)$ & $-0.0109(5)$ & $0.0039(5)$ & $-0.0035(5)$ \\
C9 & $0.0479(7)$ & $0.0250(6)$ & $0.0473(7)$ & $-0.0073(4)$ & $0.0062(6)$ & $-0.0017(4)$ \\
C10 & $0.0393(7)$ & $0.0255(6)$ & $0.0333(6)$ & $-0.0029(4)$ & $0.0091(5)$ & $-0.0004(4)$ \\
C11 & $0.0467(9)$ & $0.0209(7)$ & $0.0427(9)$ & 0.000 & $0.0075(7)$ & 0.000 \\
\hline
\end{tabular}


Geometric parameters $\left(\AA,{ }^{\circ}\right)$

\begin{tabular}{|c|c|c|c|}
\hline $\mathrm{N} 1-\mathrm{C} 1$ & $1.3345(12)$ & C6- 66 & 0.9300 \\
\hline $\mathrm{N} 1-\mathrm{C} 1^{\mathrm{i}}$ & $1.3345(12)$ & $\mathrm{C} 6-\mathrm{C} 7$ & $1.3579(15)$ \\
\hline $\mathrm{C} 1-\mathrm{H} 1$ & 0.9300 & $\mathrm{C} 7-\mathrm{H} 7$ & 0.9300 \\
\hline $\mathrm{C} 1-\mathrm{C} 2$ & $1.3840(15)$ & $\mathrm{C} 7-\mathrm{C} 8$ & $1.4158(16)$ \\
\hline $\mathrm{C} 2-\mathrm{H} 2$ & 0.9300 & $\mathrm{C} 8-\mathrm{H} 8$ & 0.9300 \\
\hline $\mathrm{C} 2-\mathrm{C} 3$ & $1.3881(12)$ & $\mathrm{C} 8-\mathrm{C} 9$ & $1.3534(16)$ \\
\hline $\mathrm{C} 3-\mathrm{C} 4$ & $1.4951(19)$ & C9-H9 & 0.9300 \\
\hline $\mathrm{C} 4-\mathrm{C} 5^{\mathrm{i}}$ & $1.4088(12)$ & $\mathrm{C} 9-\mathrm{C} 10$ & $1.4284(15)$ \\
\hline $\mathrm{C} 4-\mathrm{C} 5$ & $1.4088(12)$ & $\mathrm{C} 10-\mathrm{C} 11$ & $1.3920(13)$ \\
\hline $\mathrm{C} 5-\mathrm{C} 6$ & $1.4259(14)$ & C11-H11 & 0.9300 \\
\hline $\mathrm{C} 5-\mathrm{C} 10$ & $1.4362(16)$ & & \\
\hline $\mathrm{C} 1-\mathrm{N} 1-\mathrm{C}^{\mathrm{i}}$ & $115.86(12)$ & $\mathrm{C} 7-\mathrm{C} 6-\mathrm{C} 5$ & $121.52(10)$ \\
\hline $\mathrm{N} 1-\mathrm{C} 1-\mathrm{H} 1$ & 117.9 & $\mathrm{C} 7-\mathrm{C} 6-\mathrm{H} 6$ & 119.2 \\
\hline $\mathrm{N} 1-\mathrm{C} 1-\mathrm{C} 2$ & $124.14(10)$ & $\mathrm{C} 6-\mathrm{C} 7-\mathrm{H} 7$ & 119.6 \\
\hline $\mathrm{C} 2-\mathrm{C} 1-\mathrm{H} 1$ & 117.9 & $\mathrm{C} 6-\mathrm{C} 7-\mathrm{C} 8$ & $120.73(10)$ \\
\hline $\mathrm{C} 1-\mathrm{C} 2-\mathrm{H} 2$ & 120.2 & $\mathrm{C} 8-\mathrm{C} 7-\mathrm{H} 7$ & 119.6 \\
\hline $\mathrm{C} 1-\mathrm{C} 2-\mathrm{C} 3$ & $119.63(10)$ & $\mathrm{C} 7-\mathrm{C} 8-\mathrm{H} 8$ & 120.0 \\
\hline $\mathrm{C} 3-\mathrm{C} 2-\mathrm{H} 2$ & 120.2 & $\mathrm{C} 9-\mathrm{C} 8-\mathrm{C} 7$ & $119.95(10)$ \\
\hline $\mathrm{C} 2 \mathrm{i}-\mathrm{C} 3-\mathrm{C} 2$ & $116.60(13)$ & $\mathrm{C} 9-\mathrm{C} 8-\mathrm{H} 8$ & 120.0 \\
\hline $\mathrm{C} 2-\mathrm{C} 3-\mathrm{C} 4$ & $121.70(6)$ & $\mathrm{C} 8-\mathrm{C} 9-\mathrm{H} 9$ & 119.3 \\
\hline $\mathrm{C} 22^{\mathrm{i}}-\mathrm{C} 3-\mathrm{C} 4$ & $121.70(6)$ & $\mathrm{C} 8-\mathrm{C} 9-\mathrm{C} 10$ & $121.38(11)$ \\
\hline $\mathrm{C} 5-\mathrm{C} 4-\mathrm{C} 3$ & $119.84(6)$ & $\mathrm{C} 10-\mathrm{C} 9-\mathrm{H} 9$ & 119.3 \\
\hline $\mathrm{C} 5-\mathrm{C} 4-\mathrm{C} 3$ & $119.83(6)$ & $\mathrm{C} 9-\mathrm{C} 10-\mathrm{C} 5$ & $118.80(9)$ \\
\hline $\mathrm{C} 5-\mathrm{C} 4-\mathrm{C} 5^{\mathrm{i}}$ & $120.33(13)$ & $\mathrm{C} 11-\mathrm{C} 10-\mathrm{C} 5$ & $119.30(10)$ \\
\hline $\mathrm{C} 4-\mathrm{C} 5-\mathrm{C} 6$ & $122.77(10)$ & $\mathrm{C} 11-\mathrm{C} 10-\mathrm{C} 9$ & $121.90(11)$ \\
\hline $\mathrm{C} 4-\mathrm{C} 5-\mathrm{C} 10$ & $119.61(9)$ & $\mathrm{C} 10-\mathrm{C} 11-\mathrm{C} 10$ & $121.84(14)$ \\
\hline $\mathrm{C} 6-\mathrm{C} 5-\mathrm{C} 10$ & $117.62(9)$ & $\mathrm{C} 10^{\mathrm{i}}-\mathrm{C} 11-\mathrm{H} 11$ & 119.1 \\
\hline $\mathrm{C} 5-\mathrm{C} 6-\mathrm{H} 6$ & 119.2 & $\mathrm{C} 10-\mathrm{C} 11-\mathrm{H} 11$ & 119.1 \\
\hline
\end{tabular}

Symmetry code: (i) $-x, y,-z+1 / 2$.

Hydrogen-bond geometry $\left(A,{ }^{\circ}\right)$

$\mathrm{Cg}$ is the centroid of the pyridine ring.

\begin{tabular}{lllll}
\hline$D-\mathrm{H} \cdots A$ & $D-\mathrm{H}$ & $\mathrm{H} \cdots A$ & $D \cdots A$ & $D-\mathrm{H} \cdots A$ \\
\hline $\mathrm{C} 7-\mathrm{H} 7 \cdots C g^{\mathrm{ii}}$ & 0.93 & 2.74 & $3.5606(12)$ & 148 \\
$\mathrm{C} 7-\mathrm{H} 7 \cdots C g^{\mathrm{iii}}$ & 0.93 & 2.74 & $3.5606(12)$ & 148 \\
\hline
\end{tabular}

Symmetry codes: (ii) $-x+1,-y+1,-z+1$; (iii) $x+1,-y+1, z+1 / 2$. 